\title{
Mehr literarischer Entdecker als Polarheld: Arthur Conan Doyles Reise in die Arktis (1880)
}

\section{Martin Göllnitz}

Philipps-Universität Marburg, FB 06: Geschichte und Kulturwissenschaften, Institut für Hessische Landesgeschichte, Wilhelm-Röpke-Straße 6c, 35032 Marburg, Deutschland

Correspondence: Martin Göllnitz (mgoellnitz@uni-marburg.de)

Published: 20 May 2021

Kurzfassung. Hört man den Namen Arthur Conan Doyle, so denkt man natürlich zunächst an seinen literarischen Helden und Meisterdetektiv Sherlock Holmes, weniger an den Schriftsteller selbst. Doch nicht nur Londons Verbrechensbekämpfer Nr. 1 und sein getreuer Kompagnon Dr. John Watson haben zahlreiche Abenteuer erlebt und Gefahren getrotzt, auch deren Erfinder kann auf ein aufregendes und mitunter lebensgefährliches Unternehmen zurückblicken - das überdies vielfältige Inspirationen für dessen literarisches Schaffen bereithielt. Der vorliegende Artikel nimmt dieses Abenteuer, das Doyle im Alter von 20 Jahren als Mitglied und Schiffsarzt auf einem englischen Walfänger erlebte, in den Blick und fragt danach, wie der noch junge Medizinstudent die Arktis als Natur- und Gefahrenraum wahrnahm, von ihr beeinflusst wurde und in einem ihm fremden, sozialen Milieu agierte.

Abstract. When one hears the name Arthur Conan Doyle, one usually thinks first of his literary hero and master detective Sherlock Holmes, rather than the writer himself. But it was not only London's top crime fighter and his faithful companion (Dr. John Watson) who braved dangers, Doyle himself experienced exciting - and sometimes life-threatening - adventures, which later provided inspiration for his literary work. This article examines Doyle's journey, at the age of 20, on an English whaler as a member of the crew and ship's doctor. It further explores how the young medical student perceived, and was influenced by, the Arctic as a natural and dangerous space, as well as how he acted in this social milieu which was foreign to him.

\section{Einleitung}

Arthur Conan Doyle (Abb. 1), Sohn einer irisch-stämmigen britischen Familie von Künstlern, Karikaturisten und Staatsbeamten, der in vornehmer Armut im schottischen Edinburgh aufwuchs, war kein typischer Naturforscher der Viktorianischen Epoche, der einem Neigungsstudium nachging und im Auftrag der Royal Society die wissenschaftliche Vermessung der Welt vorantrieb. Der Sherlock Holmes-Schriftsteller, der als Medizinstudent im Alter von 20 Jahren sein Studium zugunsten einer sechs Monate dauernden Reise in die Arktis unterbrach, nahm vielmehr für sich das Bild jenes in landläufigen Klischees stets als forsch und verwegen imaginierten Polarabenteurers in Anspruch, der mit einem Bein schon im Grab, dem weißen Tod im letzten Moment von der Schippe springt. Und zwar mit allen zeitgenössischen Eigenschaften und den sich für uns heute daraus ergebenden Ambivalenzen. Denn es sind vor allem arktische Heroen wie William Baffin, William Edward Parry, Henry Hudson oder John Franklin, auf die Doyle rekurriert, wenn er rückblickend von seiner Fahrt ins Polarmeer berichtet. Vorbilder also, bei denen Entdeckung und Erkundung noch Hand in Hand gingen. Freilich unterschied sich Doyle von seinen Polarhelden erheblich, hatte er doch weder eine Karriere in der Royal Navy noch eine wirkliche Entdeckungsreise mit all den damit verbundenen widrigen Strapazen unternommen - obgleich seine Reise auf dem Walfänger keineswegs ungefährlich war.

1859 in Edinburgh geboren, hatte er nach einer erstklassigen Ausbildung in Stonyhurst, einem namhaften jesuitischen Internat in der Grafschaft Lancashire, und Feldkirch, wo er das Jesuitenkolleg Stella Matutina besuchte, 1876 mit dem Medizinstudium in seiner Heimatstadt begonnen (Lycett, 2007). Doyles Entschluss, mitten im dritten Studienjahr auf einer Walfangreise in die Arktis anzuheuern, erscheint 
auf den ersten Blick unüberlegt und waghalsig, war allerdings mit einer Reihe von seltenen Möglichkeiten verbunden. Zum einen konnte er auf diese Weise seine wachsende Abenteuerlust befriedigen, wofür er zugleich bezahlt wurde. Zum anderen bot sich ihm an Bord des Schiffes ausreichend Freiraum, um seine schriftstellerischen Ambitionen zu pflegen. So befanden sich in seiner „Seemannsausrüstung“ neben seinem Reisetagebuch auch einige Bände Poesie, Philosophie und Literatur, die ihn von den oftmals öden und wenig ereignisreichen Stunden auf See ablenken sollten (Doyle, 1989). Die Erfahrungen seiner arktischen Reise ließ er sodann in seine 1883 erstmals veröffentlichte Kurzgeschichte „The Captain of the Pole-Star" einfließen, die, verfasst in Form eines Tagesbuchs eines Medizinstudenten, der als Schiffsarzt auf einem Walfänger dient, deutlich macht, wie stark ihn die Zeit auf dem Walfänger auch schriftstellerisch beeinflusste (Doyle, 1883).

Unter den 56 Crewmitgliedern der „Hope“, deren Besatzung sich vornehmlich aus Peterhead und von den Shetlandinseln rekrutierte, stellte Doyle gewiss eine singuläre Erscheinung dar (Abb. 2). Dass es ihm dennoch gelang, sich an das Leben an Bord eines Walfängers zu gewöhnen und sich dazu in eine Gesellschaft einzufügen, die ein ihm bisher fremdes soziales Milieu bildete, lag vermutlich weniger an seinen literarischen Neigungen, als vielmehr an seiner Vorliebe für das Boxen. Ferner dürfte es für das soziale Klima an Bord zuträglich gewesen sein, dass er einerseits sein medizinisches Handwerk beherrschte, andererseits nicht davor zurückschreckte, sich aktiv an der Robbenjagd und am Walfang zu beteiligen.

Unter diesen Voraussetzungen verbrachte Doyle insgesamt sechs Monate auf dem Schiff, das am 28. Februar 1880 aus Peterhead auslief und zunächst Lerwick ansteuerte, den wichtigsten Hafen der Shetlands, von wo aus die Reise ins Polarmeer am 11. März des Jahres beginnen sollte. Erst am 11. August 1880 legte das Schiff wieder in Peterhead an, womit auch Doyles Karriere als Walfänger endete. Ein Angebot des Kapitäns, diesen auf eine zweite Reise zu begleiten, bei doppeltem Lohn als Harpunier und Schiffsarzt, lehnte er allerdings ab.

\section{Die Arktis als Naturraum}

Weniger als eine Woche nach der Abfahrt aus Lerwick stießen die Walfänger am 17. März 1880 erstmals auf offene Eisfelder, eine einmalige Erfahrung, an die sich Doyle noch Jahre später erinnerte. Was ihn dabei am meisten überraschte, war, wie schnell man die Polarregion erreichte, die ja quasi direkt vor der eigenen Haustür lag. In seinen späteren Vorträgen schilderte er seinen Zuhörern die Wirkung, die derartige Treibeisströme, losgelöst vom Packeis, auf den unerfahrenen Reisenden haben. Denn vom Moment des Erstaunens ob der Eismassen, die sich vor dem Auge des Betrachters bis ins Unendliche erstreckten, konnte auch Doyle sich nicht befreien. Um seinem damaligen Publikum - das solche Naturspektakel zumeist nur aus Erzählungen oder Reiseberichten kannte, und das nicht auf digitale Erkundungstools wie Google-Earth zurückgreifen konnte - einen Eindruck von der Größe der Eisschollen zu vermitteln, orientierte er sich zumeist an bekannten geographischen Einheiten wie englischen Grafschaften. Seine semi-wissenschaftlichen Vorträge, die er unter anderem für die „Literary \& Scientific Society“ hielt, machten ihn unter den Intellektuellen Englands rasch bekannt, sodass er zum gefragten Experten für die Arktis avancierte. Zuträglich für diese Entwicklung dürfte der Mix seiner Vorträge gewesen sein, in die er sowohl seine eigenen Erfahrungen als auch die Reiseberichte früherer Polarforscher und wissenschaftliche Analysen einfließen ließ. Die aus heutiger Sicht vermutlich fragwürdigste These, die Doyle in diesem Zusammenhang wiederholt in seinem Journal sowie in seinen Referaten oder Essays behandelte, war die zum damaligen Zeitpunkt in gelehrten Kreisen gerne diskutierte Ansicht, ,that after a certain point the temperature should change for the better as they approached the Pole" (Report on Doyle's talk „The Arctic Seas“, 1883). Gemäß dieser Theorie treibe das Eis stets in südliche Richtung, weswegen es in der Mitte einen freien Raum geben müsse. Zur naturwissenschaftlichen Bekräftigung ihrer Theorie wiesen Doyle und andere Polarforscher häufig auf die tatsächliche Form der Erde hin, die wie die übrigen Himmelskörper im Grunde keine Kugel ist, sondern eher einer Kartoffel ähnelt: Durch die tägliche Rotation der Erde um ihre Nord-Süd-Achse ist sie an den Polen abgeflacht, während sie am Äquator bauchig ist. Obgleich sie mit ihrer Annahme, die Pole seien abgeflacht, richtig lagen, zogen sie daraus die falsche Schlussfolgerung, die Pole stünden dem Erdkern wesentlich näher und deshalb sei die Temperatur dort subtropisch (Doyle, 2012).

Nach der Polarexpedition des Norwegers Fridtjof Nansen in den Jahren 1893 bis 1896 wusste aber auch Doyle, dass das Kerngebiet des Polarmeers ständig zugefroren und mit drei bis vier Meter dickem Packeis bedeckt ist (Nansen, 1898). An dessen Rändern bildet sich jedes Jahr eine ungefähr ein Meter dicke Eisschicht, von der immer wieder Schollen abbrechen, die sich dann übereinander schieben und verkeilen. Sie bilden das sogenannte Jahrespackeis. Zugleich entsteht in den Wintermonaten festes Eis an den Küsten, welches bis zu hundert Kilometer weit ins Meer hinauswächst. Im Frühling, wenn die Walfänger nach Norden segeln, bricht das feste Eis auf und treibt mit den Schollen des Jahrespackeises nach Süden.

Daneben finden sich in seinen Aufzeichnungen andere Interpretationen der arktischen Natur, wobei vor allem jene interessant erscheinen, die einen stark medizinisch ausgeprägten Bezug aufweisen (Stone, 1995). Dabei wird deutlich, dass er in seinem Reisetagebuch nur einzelne Phänomene oder Ereignisse festhielt, die er in den Folgejahren als Thesen weiter ausarbeitete. Beispielhaft soll hier ein Vorfall erwähnt werden, der im Kontext der Sichtung der ersten Treibeisströme zu verorten ist. So konnte Doyle bereits unter Deck an 


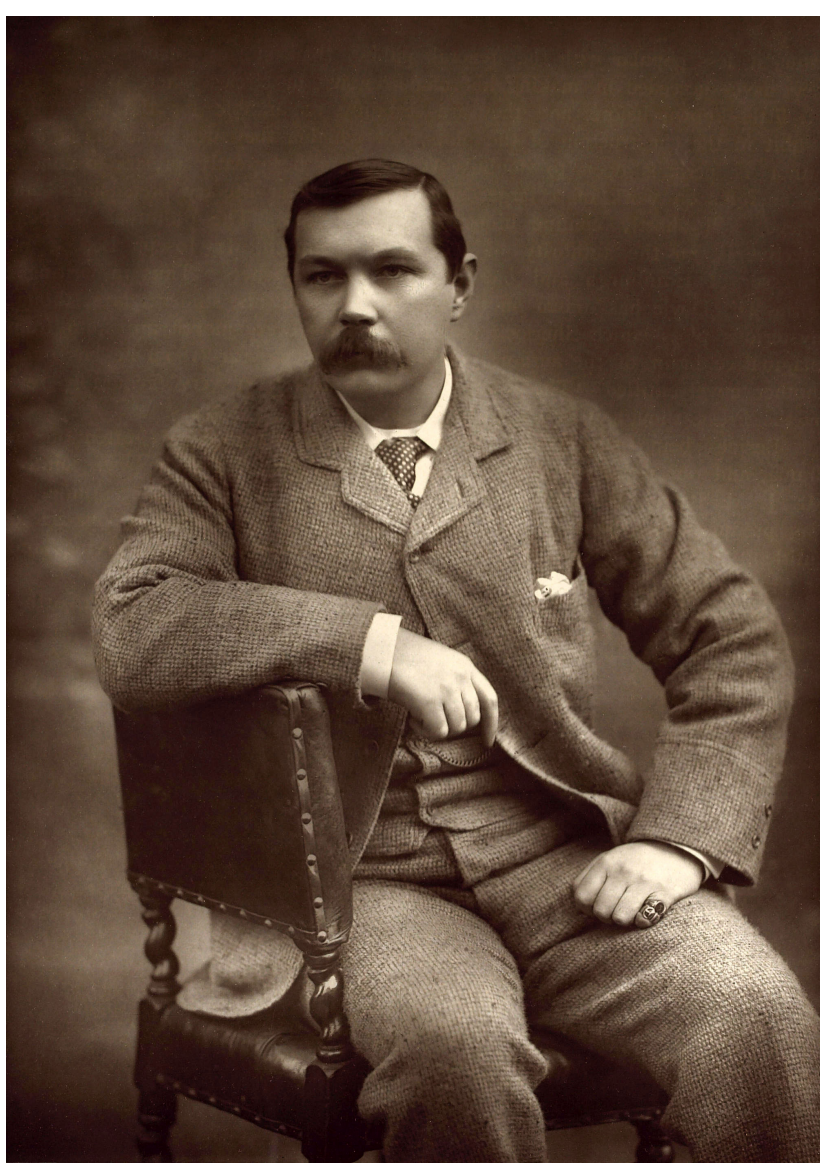

Abb. 1. Der Arzt und Sherlock Holmes-Erfinder Arthur Conan Doyle im Jahr 1893 (Fotografie von Herbert Rose Barraud, publiziert in „Men and Women of the Day 1893“, Eglington \& Co., England. Freundlicherweise zur Verfügung gestellt von der National Portrait Gallery, London.).

der scharfen, frischen Luft erkennen, dass die „Hope“ nunmehr die Polarregion und damit die Frostgrenze erreicht hatte. Aufgrund der Kälte, so seine spätere Prognose, könne kein schädlicher Keim überdauern, weshalb der arktischen Luft eine lebensspendende und heilende Wirkung zugesprochen werden müsse. In einem Interview ließ er sich sogar zu der Aussage verleiten, ,in years to come, [the Arctic] will be the world's sanatorium“ (Doyle, 1892).

\section{Die Arktis als Gefahrenraum}

Im Gegensatz etwa zu den europäischen Weltumsegelungen des 19. Jahrhunderts profitierten die Walfänger nicht von der englischen Kulturmission, die dafür Sorge trug, dass die Seefahrer und Entdecker über weite Teile der Ozeane durch Zeitungsberichte oder Briefe, die in den Häfen der europäischen Kolonien bereits auf sie warteten, über aktuelle Nachrichten und politische Entwicklungen informiert wurden. Die Walfänger waren den Großteil der Fangsaison, die sich von Fe-

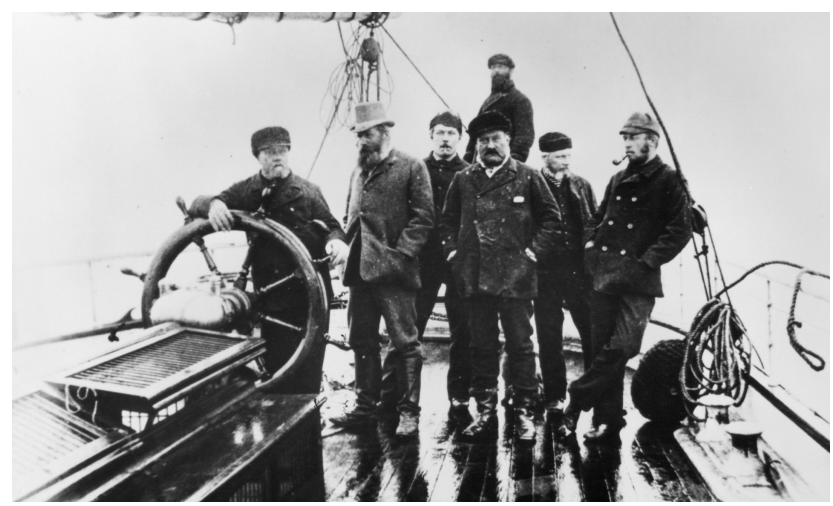

Abb. 2. Arthur Conan Doyle (3.v.1.) und Captain John Gray (4.v.1.) posieren gemeinsam mit weiteren Seemännern auf dem Achterdeck des Walfängers „Hope“ im Jahr 1880 (unbekannter Fotograf, publiziert in „The Strand Magazine“, Januar 1897).

bruar bis August erstreckte, vom Rest der „Zivilisierten“ Welt abgeschnitten. Damit einher ging eine gewisse Einsamkeit, welche auch Doyle nicht mit dem Lesen diverser Bücher oder dem Schreiben von Kurzgeschichten auszufüllen vermochte.

Um der lähmenden Totenstille und der andauernden Langeweile des Schiffsalltags zu entgehen, vor allem an jenen Tagen, an denen die „Hope“ im Eis gefangen war oder aber keine Robben beziehungsweise Wale gesichtet wurden, verbrachte Doyle seine Freizeit mit den Besatzungsmitgliedern, deren Gespräche sich vornehmlich um ,zoology, murders, executions and ironclads" drehten (Doyle, 2012). Leider geht der junge Student nicht weiter auf den Inhalt der Unterhaltungen ein. In Anbetracht von Doyles spöttischer Bemerkung, das Leben mit den Matrosen habe eine geistige Stagnation bewirkt, die sogar zu einer gewissen Verrohung beitrug, ist anzunehmen, dass es sich dabei kaum um intellektuelle Erörterungen, wie er sie aus Edinburgh kannte, gehandelt hat. Doyle kontrastierte sozusagen die wilde und raue Männlichkeit der Matrosen mit der ihm bekannten, als gebildet und zivilisiert dargestellten Männlichkeit des britischen Bürgertums. Im Gegensatz zu anderen reisenden Naturforschern seiner Zeit brandmarkte er das Verhalten der Matrosen aber nicht. Vielmehr versuchte er ihre vermeintliche emotionale Härte, aber auch die ihnen zugeschriebene starke Physis zu übernehmen.

So zeigte sich Doyle enthusiastisch was die Jagd auf Klappmützenrobben betraf, die von der Crew hingegen als ermüdend empfunden wurde. Durch seinen Eifer, so glaubte er, ermutige er als Neuling und gelehrter Außenseiter sogar seine Kameraden, und mit seinem Tatendrang signalisiere er zugleich sein Bestreben, Teil der Mannschaft zu werden. Im Vergleich zum Walfang besaß die Robbenjagd keineswegs einen sonderlich ruhmreichen Ruf, diente sie doch vornehmlich dem Zweck, die gefährliche und lange Reise zu finanzieren. Die erbarmungslose Jagd auf die ,poor little Toby's“ gewann für Doyle vor allem deshalb an Reiz, weil sie durch 
die natürlichen Gefahren der Arktis einen gewissen Kick bereithielt, wie er viele Jahre später dem irischen Schriftsteller Bram Stoker in einem Interview erzählte (Orel, 1991). Ohne Frage stellte die Robbenjagd eine lebensgefährliche Tätigkeit dar, insbesondere wenn es dem Jäger an Erfahrung auf dem Eis mangelte. Denn der Sturz ins eiskalte Wasser konnte binnen weniger Minuten den Tod bedeuten, sofern es dem Gestürzten nicht gelang, zurück aufs Eis zu klettern.

Der eigentliche Zweck der Reise, die zugleich den größten Ruhm versprach, begann im Juni 1880, als die „Hope“ in Walgebiete segelte. Da Doyle am Profit beteiligt war, hegte er ein natürliches Interesse am Erfolg der Jagd. Insgesamt verfügte das Schiff über acht Walboote, von denen im Regelfall nur sieben bemannt wurden, während die sogenannten Freiwächter (,,idlers"), sprich alle, die mit anderen Pflichten betraut waren, an Bord zurückblieben. Auf dieser Reise erklärten sich die Freiwächter, insbesondere der junge Doyle, interessanterweise dazu bereit, das achte Schiff zu bemannen und an der Waljagd teilzunehmen. Für ihn glich die lebensgefährliche Waljagd, die er in seinem Reisetagebuch äußerst lebhaft schildert, von allen ihm bekannten Herausforderungen noch am ehesten dem Mannschaftssport. Ein Aspekt, den er in Vorträgen durch die Präsentation geeigneter Tierpräparate - in Ermangelung bildhafter PowerPoint-Vorträge - unterstrich.

Insgesamt wird an seinen Beschreibungen deutlich, dass Doyle die arktische Fauna eher mit sportlichem als akademischem Interesse betrachtete und sie vornehmlich als Zielscheibe nutzte, um seine Treffsicherheit mit der Flinte oder Harpune zu beweisen. Wohl auch deshalb findet sich in seinem Journal wiederholt der Terminus ,right whale“ für den Grönlandwal, eine Bezeichnung, die er von den Walfängern übernahm, deren Interesse für zoologische Unterschiede als gering eingestuft werden muss und die damit deutlich machten, dies sei der richtige Wal zum Töten - im Gegensatz etwa zum Finnwal, der für die Segelschiffe und Ruderboote des 19. Jahrhunderts schlicht zu schnell war. Obgleich er in dieser Hinsicht äußerst kaltblütig vorging, mangelte es ihm keineswegs an Wissensdurst. Seine Einträge im Reisetagebuch lassen erkennen, dass er wiederholt sowohl die maßgebliche Fachliteratur konsultierte als auch seine Schiffskameraden in Bezug auf die Tierwelt der Arktis ausfragte. Gerne nahm er auch Anekdoten oder skurrile Begebenheiten auf, wie den seltsamen Wal mit der Eisenhaut. In weiten Teilen entsprechen seine Aufzeichnungen dabei dem Wissensstand des späten 19. Jahrhunderts, einschließlich der verbreiteten Irrtümer seiner Zeit (Göllnitz and Kragh, 2020). Die zahlreichen Beschreibungen der arktischen Flora und Fauna, die er in seinem Journal festhielt, sind trotz der naturwissenschaftlichmedizinischen Ausbildung, die er in Edinburgh genossen hatte, teilweise unzuverlässig und, dessen kuriosem schottischen Dialekt sowie dem Seemannsgarn seiner Kameraden geschuldet, auch rätselhaft.

\section{Schlussbetrachtung}

Anfang August 1880 nahmen die Walfänger wieder Kurs auf die Heimat, an Bord eine spärliche Fracht aus zwei Walen, rund 3.600 Robben und einer umfangreichen Sammlung von Eisbären, Narwalen und Polarvögeln. Aus der Sicht der Crew stellte diese Ausbeute ein eher mageres Ergebnis dar, und für sie gewann die Reise wohl keine größere Bedeutung. Doch Doyle beurteilte den Erfolg der Unternehmung an anderen Maßstäben. Zusätzlich zu der Erfahrung, der Inspiration für neue Kurzgeschichten und insgesamt 195 Pfund blieb ihm ein Foto, welches ihn auf dem Achterdeck der „Hope“ zeigt, wo er durchnässt vom Regen, unter einem grauen und drohenden Himmel, die Hände locker in die Hosentaschen gesteckt, mit kühnem Blick in den nebelverhangenen Ozean starrt (Göllnitz and Kragh, 2020). Aus dem abenteuerlustigen Medizinstudenten war ein Mann geworden. Sein einundzwanzigster Geburtstag, den er am 22. Mai 1880 nur 600 Meilen vom Nordpol entfernt vermutlich mit einer gehörigen Portion Alkohol feierte, markiert diesen Wandel. In seinem Reisetagebuch inszenierte Doyle daher überwiegend ein äußerst kühnes und wagemutiges Bild seiner neuen Männlichkeit, wenn auch zum Teil mit einem deutlichen Augenzwinkern, was sich beispielhaft an seiner unmissverständlichen Anspielung auf die heroischen Polarforscher zeigt: „I would have gone to the Pole, but my matches ran short and I couldn't get a smoke“"(Doyle, 2012).

Datenverfüg barkeit. Für diesen Artikel wurden keine Datensätze genutzt.

Interessenkonflikt. Die Autor*innen erklären, dass kein Interessenkonflikt besteht.

Begutachtung. This paper was edited by Donovan Dennis.

\section{Literatur}

Doyle, A. C.: The Captain of the Pole Star, Temple Bar, January, 33-52, 1883.

Doyle, A. C.: The Glamour of the Arctic, The Idler, July, 624-638, 1892.

Doyle, A. C.: Memories and Adventures. Nachdruck, Oxford, Oxford University Press, 1989.

Doyle, A. C.: „Dangerous Work“ Diary of an Arctic Adventure, Herausgeber: Jon Lellenberg und Daniel Stashower, The British Library, London, 2012.

Göllnitz, M. and Kragh, L.: „We've ransacked the country and taken all we could get." Arthur Conan Doyle's perception of nature and masculinity in the Arctic (1880), in: Die Arktis. Geschichte, Politik, Rezeption, Herausgeber: Alexander Friedman und Frank Jacob, Königshausen \& Neumann, Würzburg, 53-68, 2020. 
Lycett, A.: The man who created Sherlock Holmes. The life and times of Sir Arthur Conan Doyle, Free Press, New York, 2007.

Nansen, F.: In Nacht und Eis. Die norwegische Polarexpedition 1893-1896, Brockhaus, Leipzig, 1898.

Orel, H. (Hrsg.): Sir Arthur Conan Doyle: Interviews and Recollections, Macmillan, London, 1991.
Report on Doyle's talk „The Arctic Seas“, Hampshire Telegraph, December, 8, 2, 1883.

Stone, I. R.: The polar writings of Sir Arthur Conan Doyle, Polar Record, 31/176, 63-65, 1995. 